\title{
Power Scalable Video Encoding Strategy Based on Game Theory
}

\author{
Wen $\mathrm{Ji}^{1}$, Peng $\mathrm{Li}^{1}$, Min Chen ${ }^{2}$, and Yiqiang Chen ${ }^{1}$ \\ ${ }^{1}$ Institute of Computing Technology, Chinese Academy of Sciences, Beijing, China \\ ${ }^{2}$ School of Computer Science and Engineering, Seoul National University, Korea \\ \{jiwen, lipeng2008, yqchen\}@ict.ac.cn, \\ mchen@mmlab.snu.ac.kr
}

\begin{abstract}
Most mobile video applications are often operated on battery energy supply devices. The higher resolutions and complex functionality of video applications need high resource requirements. Thus, power efficient design plays an important role in computation intensive applications especially for video encoding systems. This paper proposed a power-scalable video encoding scheme (PSVE) for energy limited systems. The PSVE establishes game theoretical analysis and models the power consumption problem as a game problem. PSVE can make video encoder work under variable energy resource constraint while keep better performance. It uses game theory to solve the tradeoff between encoding effect and power consumption. Experimental results demonstrate the efficiency of the proposed approach.
\end{abstract}

Keywords: Game theory, power control, video encoding system.

\section{Introduction}

With the rapid expansion of mobile multimedia applications, efficient use of energy resource grows in more importance. In most cases, video applications often operate on the mobile devices or terminals with limited battery capacity. A urgent requirement for portable wireless video systems is low power dissipation. Thus, power control play important roles in these systems. In video encoding systems, designing the energy aware systems so as to extend the battery lifetime is an effective way. Video encoding systems need flexible tradeoffs between encoding quality and power consumption. [1] presents a power aware video encoding system by embedding some reconfigurable points inside so as to provide system-level power-aware control. It gives the sufficient condition of a poweraware encoding design. From the tradeoff between encoding effect and power consumption aspect, Power-Rate-Distortion (P-R-D) model [2] is a widely referenced method. It analyzes the rate-distortion (R-D) behavior of video encoding system under the energy constraint. Based on this, further research in 3 proved power is tightly coupled with rate, thus map bits to joules to perform energy minimization is rapid method to obtain the minimum energy. These provide the theoretical analysis for power scalable video encoding. Scalable video codec is studied in [4. It considers the memory, processing power, and bridge these with

P. Muneesawang et al. (Eds.): PCM 2009, LNCS 5879, pp. 1237-1243, 2009.

(C) Springer-Verlag Berlin Heidelberg 2009 
amount of bandwidth which comes from video fragment. Thus, energy consumption scalable video encoding becomes a tendency especially in energy constraint applications. Recently, game theory has been introduced to wireless and mobile applications and has been shown to become an effective tool for solve power control problems. Newly, game theory has been recognized as an emerging solution for rate control optimization in video coding systems [5]. Game theory is the study of multi-player decision problems. A well known property from game theory is the equilibrium-optimization based on notions of fairness or efficiency and mathematic characteristics [6].

In this paper, we proposed an power-scalable video encoding scheme for energy constraint terminals and investigated the Nash equilibrium in game theory. Since this scalable power control in video encoding systems has multiple equilibria so as to maintain low power consumption in each scalable level, we then modeled the rate-distortion-complexity (RDC) behaviors of the key modules in video encoder. Finally, a scalable and low power control video encoding system based on Nash equilibrium solution is obtained.

This paper is organized as follows. After briefly reviewing, section 2 gives the game theoretic formulation addressed in Section 1 and contains the main contribution of the paper: A variety of distributed algorithms for the computation of the Nash equilibria of the encoding modules; Section 3 gives the results in video encoding systems; and Section 4 concludes.

\section{System Model and Problem Formulation}

Our goal includes two parts, one is to provide a scalable power control encoder so as to adapt the system resource; the other is to find a good configuration that has optimal visual quality and optimal rate under power constraint. In practice, there are various parameters that can be adjusted to lower the power consumption in a video encoder [1. A basic video encoder consists of finite functional modules: motion estimation, prediction, DCT, entropy coding and so on. Then the encoder can be regarded as a finite modules set, and these modules can be analyzed from basic RDC relationship. Then, when an encoder provides power scalable output under the power consumption budget, it is decomposed to the following steps:

\subsection{Power Budget Mapping and Computing}

This module includes two parts. Part 1, user can specify the working states of video service. These states include 'Maximum battery time mode', 'Battery optimized mode', 'Maximum performance mode' and so on. Each state corresponds to a battery working mode of the device. These states are widely used in mobile devices and terminals. Part 2, to automatically adapt the working state of video service according to the perception of remaining battery capacity. For instance, 'Maximum battery time mode' can be used when the residual capacity is under $30 \%$ while 'Maximum performance mode' is adopted when the capacity is above 
$90 \%$. If the result of part 1 and part 2 do not matched, i.e. user configure the device as 'Maximum performance mode' but the residual capacity is under $30 \%$, the final available power consumption profile is based on perceptual remaining battery capacity. That is to say part 2 has a higher priority than part 1, and user can manually specify the working state only when the device resource is sufficient. Thus, this module implements a mapping and computing bridge between the power profile and the device available resource.

\subsection{Game Method in Power Control Video Encoding System}

Advanced video coding standards including MPEG-4, H.264, AVS [7] are based on the same generic design. It has been commonly recognized that the most computation consumed comes from the major modules including motion estimation (ME), discrete cosine transform (DCT), deblocking filter, mode decision (MD), and entropy coding. Module analysis based on H.264 are provided in [8] 9] for details. For the integrity, the following RDC analysis of these modules is based on the AVS standard, the video coding standard developed by the Audio and Video Coding Standard Workgroup of China, which promises similar performance but lower complexity compared with H.264. And the conclusion from this discussion is easy applied or transferred to the other video standards such as H.264, MPEG2, etc.

Because the encoding process can be regarded as the aggregation of modules. Each module is also under common resource-constrained and is a competitor of battery energy for others. Ideally, a module unit would like to achieve better encoding quality while it expends a small amount of energy. Thus, it is possible to consider both encoding effect and power consumption as commodities that a module desires. Hence, finding a good balance between the two conflicting objectives is the primary focus on power control video encoding systems.

There are various configurations that can be adjusted to lower the power consumption in each module. Consequently, power control scheme in video encoding systems is transferred to do decisions under certain conditions among these modules, and finally receive some benefit or tradeoff under energy resource constraint. Modules compete in limited resource and each of them expects enough resource to achieve better performance. Then, games are characterized by the modules. Game theory is the appropriate framework for modeling such a strategic situa-

tion. Each module is regarded as a player. These players compete for the use of a fixed power resource, which is the power consumption budget. Thus, we propose a non-cooperative power allocation game, in which each module seeks to choose its encoding power over whole budget to maximize its overall utility. The concept of utility is commonly used in economics and refers to the level of satisfaction the decision-taker receives as a result of its actions. A utility function for each player reflects its preference [10].

\subsection{Utility Function in Power Control Game}

For the sake of clarity, in the following discussion, we analyze the general advanced video codec from four aspects, which represent main adjusting module configurations in encoder. 
Consider the encoding system where each module using $p_{i}$ watt of power consumption. Let $q_{i}$ denote the PSNR of the module effect on video encoder and $r_{i}$ denote the coded bit rates of the module effect. These parameters depend on the behavior analysis of the modules in the encoding systems including motion estimation, motion vector resolution, inter prediction, intra prediction and so on 8]. Utility theory helps us to build the preference relations. The utility function can be expressed as

$$
u_{i}\left(q_{i}, r_{i}, p_{i}\right)=f\left[f_{Q}\left(q_{i}\right), f_{R}\left(r_{i}\right), f_{P}\left(p_{i}\right)\right]
$$

where $f$ is the result of rate-distortion-complexity (RDC).

- Modeling the behavior of each module: For a given codec, the four module behaviors cover nearly the main features of computation and memory complexity, and the distortion introduced by the above behaviors are determined by adjusting encoding parameters. Thus, these behaviors is estimated by the statistic distributions based on typical video sequences. Through the collection of the bitrate, distortion, and computational cost, the statistic distributions of the behavior parameters can be obtained.

1) search range $(\mathrm{SR})$ : to search for the best matched $\mathrm{MB}$, motion estimation search, which search for all possible candidates in a SR, can guarantee the Sum of Absolute Difference (SAD) value, but the complexity of exhausted search is the highest. As mentioned in [9], increasing both reference frame numbers and search size leads to higher access frequency, up to approximately 60 times, while it has a minimal impact on PSNR and bit rate performances. Thus, SR reflects the computation consumption of video codec under the rule of SAD.

2) motion vector resolution (MVR): the accuracy of motion vectors has three cases, which are integer-pixel, fractional pixel in $1 / 2$, and $1 / 4$. For the encoder, $1 / 2$ pixel search results in a serious increase of access frequency processing time, and $1 / 4$ pixel accuracy increases the processing time about $10 \%$ when reduces the bit-rates up to $30 \%$ 9]. MVR is regarded as an important behavior feature for nearly all ME algorithms.

It is quite common that new techniques used in video encoder, such as the spatial prediction in intra mode coding, variable block size in inter mode decision, leads to the increase in computational complexity. In AVS, there are four different block sizes in inter mode decision, and five modes in intra mode decision. Thus, to achieve the best encoding effect, the encoder tries all the possible modes to select the best matched. This method actually costs high computational complexity so that it limits the use of video encoders in resource constraint applications. The key problem of MD module is how to adaptively select the candidate modes of this module. The complexity increases as the number of candidate modes increasing. It is possible to reduce the computational complexity without sacrificing the R-D performance by using only a few modes in MD modules [1]. Then the behavior of MD modules come up to two points: 1) providing a suitable MD output set by controlling the number of candidates; 2 ) trying to make the actual best matched mode in the candidates. 
3) Intra mode decision set: The combination of possible intra predictions. The number of candidate intra mode is $k$, (i.e $k=5$ in AVS and $k=9$ in H.264). The number of set elements is $\sum_{i=1}^{k} C_{k}^{i}$.

4) Inter mode decision set: Following the definition of set 3 ), this set is the combination of possible inter predictions. Given $k$ candidate inter mode, (i.e $k=4 \mathrm{in}$ AVS). The number of set elements is $\sum_{i=1}^{4} C_{4}^{i}$. The possible value is the combination of the four different block sizes $(16 \times 16,16 \times 8,8 \times 16,8 \times 8)$.

For the sake of simplicity, we use weight linear form to make equal (1) into practice. That is,

$$
u=u_{S R}+u_{M V R}+u_{\text {inter } M D}+u_{\text {intraMD }}
$$

Furthermore, from the curve fitting of the RDC analysis, we can get the expression of the $u_{i}$ components in equal(2).

\subsection{Non-cooperative Power Control Game in Video Encoding System}

Using game theory, we can get the non-cooperative power control game $G=$ $\left[P_{i}, u_{i}\right]$, where $i \in[1, N]$ and $N$ represents the max number of module in the game. In this game, $N=4$. Thus we have the modules' power allocation strategy set $\left\{P_{i}\right\}$ and the payoff function $u_{i}$ for $i$ th module. Each module selects a power level $p_{i}$ and $p_{i} \in P_{i}$. Let the power vector $\mathbf{p}=\left(p_{1}, \ldots, p_{N}\right) \in \mathbf{P}$ denote the outcome of the game in terms of the selected power levels of all the modules, where the $\mathbf{P}$ is the set of all power vectors. The resulting utility level for the $i$ th module is $u_{i}(\mathbf{p})$. Use this notation, $\mathbf{p}_{-i}$, alternative, is the combination of the power vectors except player $i$. This notation emphasizes that the $i$ th module controls only its own power $p_{i}$. Accordingly, the strategy space of all the modules excluding the $i$ th module is denoted by $\mathbf{p}_{-i}$. Thus the utility $i$ th module obtained by consumption $p_{i}$ can be re-expressed as $u_{i}\left(p_{i}, \mathbf{p}_{-i}\right)$.

As mentioned above, we use curve fitting from RDC analysis to re-analysis the encoding process, consequently, guarantee the strategy space $\mathbf{P}_{i}$ of each module is a compact, convex set with minimum and maximum power consumption constraints denoted by $\mathbf{P}_{i} \in\left[P_{\min }, P_{\max }\right]$. Then we can obtain the Nash Equilibrium point [12], which should be exist and unique. Then for power-scalable control, the non-cooperative power control encoding game can be developed by solving the utility function in each power consumption level. The problem of obtaining the optimal power allocation scheme in each power level can be transferred into finding a solution to maximize the modules' utility in a distributed fashion in non-cooperative power control game. It can be expressed as

$$
\max _{p_{i} \in \mathbf{P}_{i}} u_{i}\left(p_{i}, \mathbf{p}_{-i}\right), i \in[1, N]
$$

Here, the best playoff results in the best response from the other players. Thus, the optimized solution can be obtained by using Cournot approach. As is known, Cournot solution [6] has been widely discussed and accepted in equilibrium 
solution. Hence we use its main feature to solve the power equilibrium in moduleslevel power control. We get

$$
\left\{\begin{array}{l}
\frac{\partial u_{i}}{\partial p_{i}}=0 \quad i=1, N \\
\max \sum_{i=1}^{N}\left(u_{i}\right) \quad \text { s.t. } \sum_{i=1}^{N} p_{i} \leq P_{\text {budget }}^{\text {level }(j)}
\end{array}\right.
$$

Here $P_{\text {budget }}^{\text {level }(j)}$ represents the power consumption budget in level $j$. Here, $N=4$.

\section{Solution and Results}

In this section, we evaluate the performance when applying our PSVE scheme in SR, MVR, inter MD and intra MD modules. We compare the performance of PSVE with a rapid power control scheme (RPC). RPC scheme decides a encoding state in each module rapidly and configure the encoder parameters according to the given power budget. Both PSVE and RPC work under the same power consumption budget so as to obtain fair evaluation. We also tested the scalable effect. We give the results when the two encoders work in the situations of $20 \% \sim 100 \%$ of power budget. We test the performance of PSVE in four situations. In Fig. 1, the solid lines represent the relationship between encoding effect and computational cost of the experimental results which used PSVE to decide the best working states, the dashed lines, represent the results in which $\mathrm{RPC}$ is used. The quantization parameter (QP) is equal to 16. Take the news CIF sequence for example, the PSVE can make the encoder work with lowest computational consumption, while have the best encoding performance. PSVE can scale the encoder into different working state, with small PSNR variety and different computational cost level. The encoder in PSVE always works under low cost consumption while keeps better encoding effect, because the PSVE can help the modules in encoder to choose the best configurations under cost consumption budget. In each sequence test, the encoding effect which adopted PSVE is better than the one not used PSVE. Generally, Fig. 1 shows that PSVE can make the encoder have power scalable output, at the same time maintain best encoding effect which includes better video quality and lower bit rare in each scalable mode.
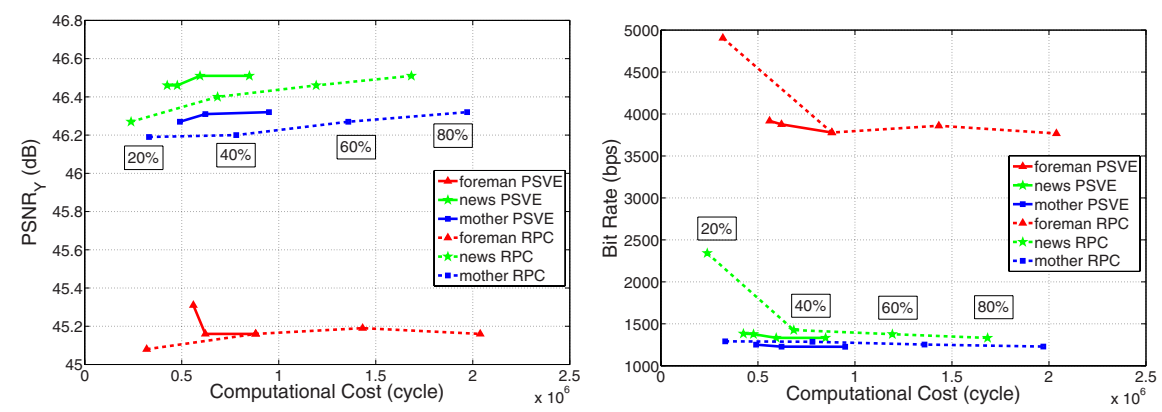

Fig. 1. PSVE performance: decision results on encoding effect of three sequences 


\section{Conclusion and Future Work}

This paper proposed PSVE strategy in power control video encoding systems. There are two major contributions in this work. First, video encoder can work under variable energy resource limitation marked with different power consumption budgets; Second, game theory is adopted to solve the tradeoff between encoding effect and power consumption, so as to obtain better performance in each consumption levels. In fact, PSVE will be fine scalable when introduce more modules. Of course, the degree of lower power consumption lies in not only PSVE but also the algorithm of each modules. But PSVE can help the encoder provide many low power working mode while keep better performance, whatever the algorithm of modules will be designed. Therefore, we will expand and practice this method into more encoding modules so that finely scale the power consumption levels in future research.

\section{References}

1. Chung Jr., L., et al.: Power-Aware Multimedia: Concepts and Design Perspectives. IEEE Circuits and Systems Magazine 7(2), 26-34 (2007)

2. He, Z., et al.: Power-Rate-Distortion Analysis for Wireless Video Communication Under Energy Constraints. IEEE CSVT 15(5) (May 2005)

3. He, Z., et al.: Energy Minimization of Portable Video Communication Devices Based on Power-Rate-Distortion Optimization. IEEE CSVT 18(5) (May 2008)

4. De Schrijver, D., et al.: MPEG-21 bitstream syntax descriptions for scalable video codecs. Multimedia Systems 11(5), 403-421 (2006)

5. Ahmad, I., Luo, J.: On Using Game Theory to Optimize the Rate Control in Video Coding. IEEE CSVT 16(2), 209-219 (2006)

6. Rasmusen, E.: Games and Information: An Introduction to Game Theory, 3rd edn. Blackwell Publishers, Malden (2001)

7. Gao, W., Reader, C., et al.: AVS-The Chinese Next-Generation Video Coding Standard, http://www.avs.org.cn/reference.asp

8. Marpe, D., et al.: The H.264/MPEG4 Advanced Video Coding Standard and its Applications. IEEE Comm. Magazine 44(8), 134-143 (2006)

9. Ostermann, J., et al.: Video coding with H.264-AVC: Tools, Performance, and Complexity. IEEE Circu. Sys. Magazine 14(1), 7-28 (2004) (First Quarter)

10. Ferguson, T.S.: Game theory. Class Text for University of California at Los Angeles (2000)

11. Su, L., Lu, Y., et al.: Complexity-Constrained H.264 Video Encoding. IEEE CSVT 19(4), 477-490 (2009)

12. Nash, John: Non-Cooperative Games. The Annals of Mathematics 54(2), 286-295 (1951) 